\title{
An algorithm for generating quasiperiodic patterns and their approximants ${ }^{\dagger}$
}

\author{
E A LORD, K RAMAKRISHNAN and S RANGANATHAN* \\ Department of Metallurgy, Indian Institute of Science, Bangalore 560 012, India
}

\begin{abstract}
An algorithm for projecting the interior of a hypercube in $\boldsymbol{N}$-dimensions on to an $\boldsymbol{m}$-dimensional subspace has been developed and incorporated into a computer program for generating quasiperiodic and periodic patterns in an $\boldsymbol{n}$-dimensional subspace. Some aspects of the resulting orthorhombic approximants to Penrose tiling patterns are discussed and illustrated.
\end{abstract}

Keywords. Projection method; quasiperiodic; tiling patterns; approximants.

\section{Introduction}

Since the discovery of quasicrystals (Schechtman et al 1984; Levine and Steinhardt 1984) very many figures have appeared in the literature illustrating quasiperiodic tilings of the plane and their periodic approximants. An elegant general method for generating quasiperiodic tiling patterns consisting of a finite number of types of rhombic (in higher dimensions, generalized rhombohedral) tiles is the projection method (Kramer and Neri 1984; Conway and Knowles 1986). The details of the algorithms that we have used to obtain these patterns are rarely discussed in the literature. A presentation of a general algorithm for projecting a strip of an $N$-dimensional hypercubic lattice on to an $n$-dimensional subspace has been described by Vogg and Ryder (1996).

A tiling of the plane by rhombuses, that has longrange orientational order exhibiting $N$-fold symmetry, is obtained by projecting a 'strip' of a hypercubic lattice (in $N$ dimensions if $N$ is odd or in $N / 2$ dimensions if $N$ is even) on to a plane perpendicular to the $[11 \ldots 1]$ axis. The two-dimensional (square) facets of the hypercubes project to rhombic tiles in the plane (figure 1). The cases $N=5,8$ and 12 are of particular interest because of their relevance to the structural modeling of decagonal, octagonal and dodecagonal quasicrystalline alloys. As is wellknown, the tilings of three-dimensional space by two kinds of rhombic hexahedral tiles arises from the $N=6$, $n=3$ case, and are related to the structure of icosahedral phases (Katz and Duneau 1986). The interesting case of $N=8, n=4$ has been discussed by Sadoc and Mosseri (1988). (The lattice in 8-dimensional space in this case is a sublattice of the hypercubic lattice, viz. the $E_{8}$ latticesee, for example, Conway and Sloane 1988).

\footnotetext{
*Author for correspondence

${ }^{\dagger}$ Paper presented at the 5th IUMRS ICA98, October 1998, Bangalore
}

\section{Projection of a hypercubic lattice}

The $2^{N}$ vertices of a unit hypercube in $E_{N}$, centred at the origin, are given by the position vectors

$$
\frac{1}{2}\left( \pm \mathbf{r}_{1} \pm \mathbf{r}_{2} \ldots \pm \mathbf{r}_{N}\right)
$$

where the vectors $\mathbf{r}_{\mathrm{i}}(\mathrm{i}=1, \ldots N)$ are the columns of an orthogonal matrix $R$ :

$$
R R^{T}=I
$$

The matrix $R$ can be split into an $n \times N$ matrix $A$ and an $m \times N$ matrix $B(m=N-n)$ :

$$
R=\left(\begin{array}{l}
A \\
B
\end{array}\right) .
$$

The columns $\mathbf{a}_{\mathrm{i}}(\mathrm{i}=1, \ldots N)$ of $A$ span an $n$-dimensional subspace $E_{\|}$and the columns $\mathbf{b}_{\mathrm{i}}(\mathrm{i}=1, \ldots N)$ of $B$ span an $m$-dimensional subspace $E_{\perp}$. These two spaces are orthogonal to each other. The images of the vertices of the hypercube, under orthogonal projection on to $E_{\perp}$, are given by

$$
\frac{1}{2}\left( \pm \mathbf{b}_{1} \pm \mathbf{b}_{2} \ldots \pm \mathbf{b}_{N}\right)
$$

The interior of the hypercube projects to a region $W$ in $E_{\perp}$ bounded by $(m-1)$-dimensional rhombohedra (the images of $(m-1)$-dimensional facets of the $N$-dimensional hypercube). The projection method for producing a tiling of $E_{\|}$ can be described briefly as follows: project on to $E_{\perp}$ the points of the hypercubic lattice defined as the set of all points with position vectors of the form

$$
\frac{1}{2}\left(x_{1} \mathbf{r}_{1}+x_{2} \mathbf{r}_{2}+\ldots+x_{N} \mathbf{r}_{N}\right)+\mathbf{a}
$$


with integer coefficients $x_{\mathrm{i}}(\mathrm{i}=1, \ldots N)$. (a is a fixed vector, corresponding to a translational shift of the lattice relative to the origin). The images are the points of $E_{\perp}$ with position vectors of the form

$$
\mathbf{x}=\frac{1}{2}\left(x_{1} \mathbf{b}_{1}+x_{2} \mathbf{b}_{2}+\ldots+x_{N} \mathbf{b}_{N}\right)+\mathbf{y},
$$

( $\mathbf{y}$ is a fixed vector in $E_{\perp}$, the projected image of a). The region $W$ (the 'window' region) provides a selection rule: Only those lattice points that project to points inside $W$ are orthogonally projected to $E_{\|}$. The resulting set of points in $E_{\|}$are, in general, the vertices of a tiling pattern in $E_{\|}$, the tiles being projected images of $n$-dimensional hypercubes. (We say 'in general' in order to avoid having to discuss singular cases.)

The method outlined above is, of course, very well known. Clearly, the central problem to be solved in producing a practical algorithm for computing the patterns in $E_{n}\left(=E_{\|}\right)$is that of determining the window region $W$.

\section{Properties of the hypercube}

A hypercube in $E_{N}$ has $2^{N-j}\left(\begin{array}{c}N \\ j\end{array}\right) j$-dimensional 'facets' (i.e. it contains $2^{N-j}\left(\begin{array}{c}N \\ j\end{array}\right) j$-dimensional hypercubes). They occur in $\left(\begin{array}{c}N \\ j\end{array}\right)$ sets of $2^{N-j}$ parallel facets. For example, in $E_{3}$ a cube has 3 sets of 4 parallel edges and 3 pairs of parallel faces; in $E_{4}$ a hypercube has 4 sets of 8 parallel edges, 6 sets of 4 parallel plane faces and 4 pairs of parallel cubic cells. When $E_{N}$ is projected on to an $m$-dimensional subspace $E_{\perp}$, a hypercube centred at the origin projects to a region bounded by $\left(\begin{array}{c}N \\ m-1\end{array}\right)$ pairs of parallel hyperplanes. The

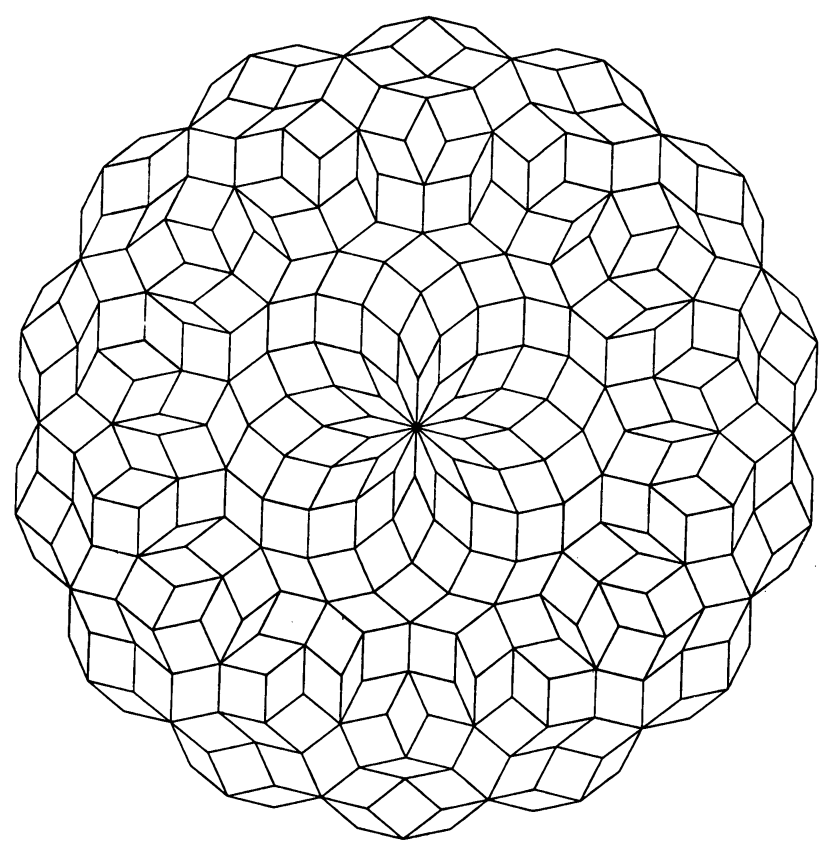

Figure 1. The $N=7, n=2$ case with $\mathbf{y}=0$. Quasiperiodic tiling by three types of rhombuses. central problem to be solved is therefore to identify the two hyperplanes, out of each set of $2^{N-m+1}$ parallel hyperplanes, that are furthest from the origin of $E_{\perp}$.

\section{Algorithm to obtain $W$ as a set of inequalities}

We shall indicate the approach adopted in our algorithm by an illustrative example: the simple case of $N=3$, $n=1, m=2$ (a cube in $E_{3}$ projected on to a plane). The region $W$ is, in general, a hexagon with pairs of opposite edges equal and parallel).

In figure 2 the projected vertices of the cube are labeled by sets of integers, which indicate the number of minus signs in the expression (4) for the position vector of a vertex. We compute the equations of the four parallel lines

$01,212,313,23123$.

Any line that does not pass through the origin has an equation of the form

$$
\mathbf{x} \cdot \mathbf{q}=1
$$

and its distance from the origin is

$$
d=1 /|\mathbf{q}|
$$

The coordinates of the points marked 0 and 1 in figure 2 have position vectors

$$
\begin{aligned}
& \mathbf{v}_{1}=\mathbf{b}_{1}+\mathbf{b}_{2}+\mathbf{b}_{3}, \\
& \mathbf{v}_{2}=-\mathbf{b}_{1}+\mathbf{b}_{2}+\mathbf{b}_{3},
\end{aligned}
$$

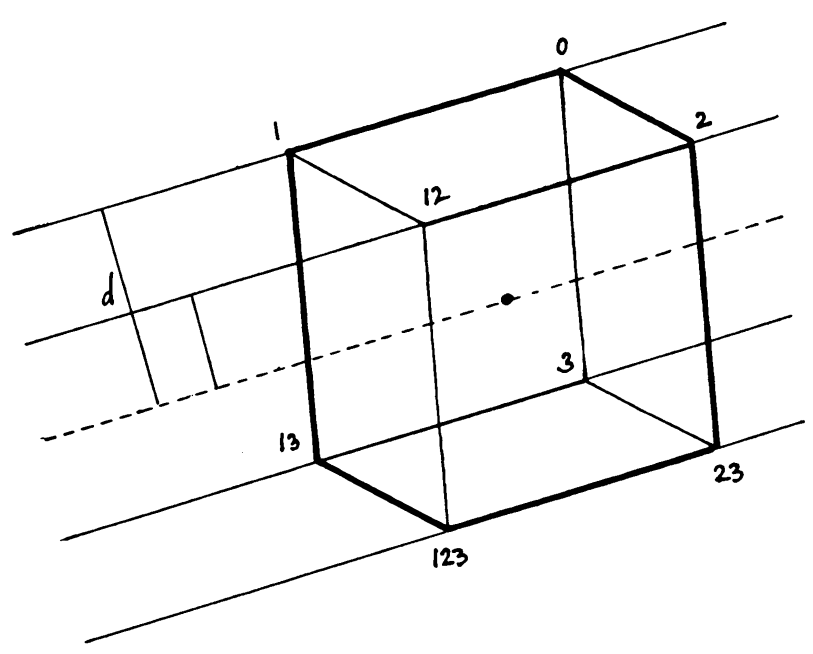

Figure 2. General projection of a cube on to a plane. 
(for convenience we drop the factor $1 / 2$ from (4) - the cube is taken to be a cube of edge length 2). Hence the line 01 is given by (7) with q satisfying

$$
\mathbf{v}_{1} \cdot \mathbf{q}=\mathbf{v}_{2} \cdot \mathbf{q}=1
$$

(If there is no solution this means that the line passes through the origin and so, obviously, cannot be part of the boundary of $W$.) Next, we consider the line 212 parallel to the previous one. Its two vertices are

$$
\begin{aligned}
& \mathbf{v}_{\mathbf{3}}=\mathbf{b}_{1}-\mathbf{b}_{2}+\mathbf{b}_{3}, \\
& \mathbf{v}_{\mathbf{4}}=-\mathbf{b}_{1}-\mathbf{b}_{2}+\mathbf{b}_{3} .
\end{aligned}
$$

If a $\mathbf{q}$ is known for the previous line, there is no need to solve the equations, because we know that the vectors $\mathbf{q}$ for parallel lines differ only by a scalar factor. Hence, we can take the previous $\mathbf{q}$ and compute $k=\mathbf{v}_{3}$.q. If $k>1$ this means that the line currently under consideration is further from the origin than the previous line, so we can simply replace: $\mathbf{q} \rightarrow \mathbf{q} / k$. Otherwise, $\mathbf{q}$ remains unchanged. Proceeding in this way, we find the vectors $\pm \mathbf{q}$ for the two lines from the set of four parallel lines that are furthest from the origin and therefore form part of the boundary of $W$.

We proceed similarly for the other quartets of parallel lines

$02,121,323,13123$ and $03,113,223,12123$.

We then have three vectors $\mathbf{q}_{1}, \mathbf{q}_{2}$ and $\mathbf{q}_{3}$ which define the hexagonal region $W$ as a set of inequalities

$$
-\frac{1}{2}<\mathbf{q} \cdot(\mathbf{x}+\mathbf{y})<\frac{1}{2}
$$

In higher dimensions the analogue of (10) is a set of $m$ linear equations in $m$ unknowns $q_{\mathrm{i}}(\mathrm{i}=1, \ldots m)$; in matrix notation,

$$
V \mathbf{q}=\mathbf{j}
$$

where $\mathbf{q}$ is $m \times 1, V$ is $m \times m$ and $\mathbf{j}$ is a column of $m 1 s$. In our implementation of the algorithm we employed an iterative algorithm (Lord et al 1990) for solving these equations.

\section{Orthorhombic approximants of Penrose tiling patterns}

The standard rotation matrix for producing quasiperiodic tilings of the plane by Penrose rhombs from a hypercubic lattice in $E_{5}$ is

$$
\frac{1}{\sqrt{10}}\left(\begin{array}{ccccc}
2 & -\sigma & -\tau & -\tau & -\sigma \\
0 & \tau \beta & \beta & -\beta & -\tau \beta \\
2 & -\tau & -\sigma & -\sigma & -\tau \\
0 & \beta & -\tau \beta & \tau \beta & -\beta \\
\sqrt{2} & \sqrt{2} & \sqrt{2} & \sqrt{2} & \sqrt{2}
\end{array}\right),
$$

where $\tau=(1+\sqrt{ } 5) / 2, \sigma=(1-\sqrt{ } 5) / 2$ and $\beta=\sqrt{ }(3-\tau)$.

Since linear deformations of the space $E_{\perp}$ will not affect the pattern produced in $E_{\|}$, it is legitimate to eliminate any overall factor in any row of $B$, so for simplicity we may take

$$
B=\left(\begin{array}{ccccc}
2 & -\tau & -\sigma & -\sigma & -\tau \\
0 & 1 & -\tau & \tau & -1 \\
1 & 1 & 1 & 1 & 1
\end{array}\right)
$$

A periodic pattern consisting of the same pair of rhombic tiles as those of the quasiperiodic patterns is obtained if the elements of the matrix $B$ are replaced by rational numbers. It is well known that periodic approximants of icosahedral and decagonal quasicrystals correspond to the replacement of the golden number $\tau$ by $p / q$, where $q$ and $p$ are successive terms in the Fibonacci sequence

$$
\begin{array}{lllllllll}
1 & 1 & 2 & 3 & 5 & 8 & 13 & \ldots
\end{array}
$$

The periods in the pattern produced in $E_{\|}$arise because, with rational numbers as components of the vectors $\mathbf{b}_{\mathrm{i}}$ $(i=1, \ldots 5)$, we will get identities of the form

$$
x_{1} \mathbf{b}_{1}+x_{2} \mathbf{b}_{2}+\ldots+x_{5} \mathbf{b}_{5}=0
$$

with integer coefficients $x_{\mathrm{i}}(\mathrm{i}=1, \ldots 5)$. Whenever this occurs, the vector

$$
x_{1} \mathbf{a}_{1}+x_{2} \mathbf{a}_{2}+\ldots+x_{5} \mathbf{a}_{5}
$$

will be a period for the pattern in $E_{\|}$.

An ambiguity arises because

$$
\sigma=-\tau^{-1}=1-\tau
$$

so that a reasonable approximant for $\sigma$ could be either $-q / p$ or $1-p / q$. We thus have two possible schemes. In addition, we can use two different Fibonacci approximants for $\tau$, replacing $\tau$ by $p_{1} / q_{1}$ in the first row of $B$ and by $p_{2} / q_{2}$ in the second row. We may call the resulting pattern a $p_{1} / q_{1}, p_{2} / q_{2}$ approximant.

Scheme I: $B=\left(\begin{array}{ccccc}2 p_{1} q_{1} & -p_{1}^{2} & q_{1}^{2} & q_{1}^{2} & -p_{1}^{2} \\ 0 & q_{2} & -p_{2} & p_{2} & -q_{2} \\ 1 & 1 & 1 & 1 & 1\end{array}\right)$, 
Scheme II: $B=\left(\begin{array}{ccccc}2 q_{1} & -p_{1} & p_{1}-q_{1} & p_{1}-q_{1} & -p_{1} \\ 0 & q_{2} & -p_{2} & p_{2} & -q_{2} \\ 1 & 1 & 1 & 1 & 1\end{array}\right)$,

For scheme I the periods are

$$
\begin{aligned}
& 2\left(p_{1}^{2}+q_{1}^{2}\right) \mathbf{a}_{1}-\left(2 p_{1} q_{1}+p_{1}^{2}\right)\left(\mathbf{a}_{3}+\mathbf{a}_{4}\right)+\left(2 p_{1} q_{1}-q_{1}^{2}\right) \\
& \left(\mathbf{a}_{2}+\mathbf{a}_{5}\right) ; q_{2}\left(\mathbf{a}_{3}-\mathbf{a}_{4}\right)+p_{2}\left(\mathbf{a}_{2}-\mathbf{a}_{5}\right) .
\end{aligned}
$$

For the Fibonacci approximants we set

$$
p_{1}=F_{n_{1}}, q_{1}=F_{n_{1}-1}, p_{2}=F_{n_{2}}, q_{2}=F_{n_{2}-1} .
$$

The identities

$$
\begin{aligned}
& p \tau+q=\tau^{n}, \mathbf{a}_{3}+\mathbf{a}_{4}=-\tau \mathbf{a}_{1}, \mathbf{a}_{2}+\mathbf{a}_{5}=(\tau-1) \mathbf{a}_{1}, \\
& \mathbf{a}_{2}-\mathbf{a}_{5}=\tau\left(\mathbf{a}_{3}-\mathbf{a}_{4}\right),
\end{aligned}
$$

then lead to the following expressions for the periods:

$$
\begin{gathered}
\tau^{2 n-1} \sqrt{5} \mathbf{a}_{1} \\
\tau^{n}\left(\mathbf{a}_{3}-\mathbf{a}_{4}\right)
\end{gathered}
$$

We thus get orthorhombic unit cell with

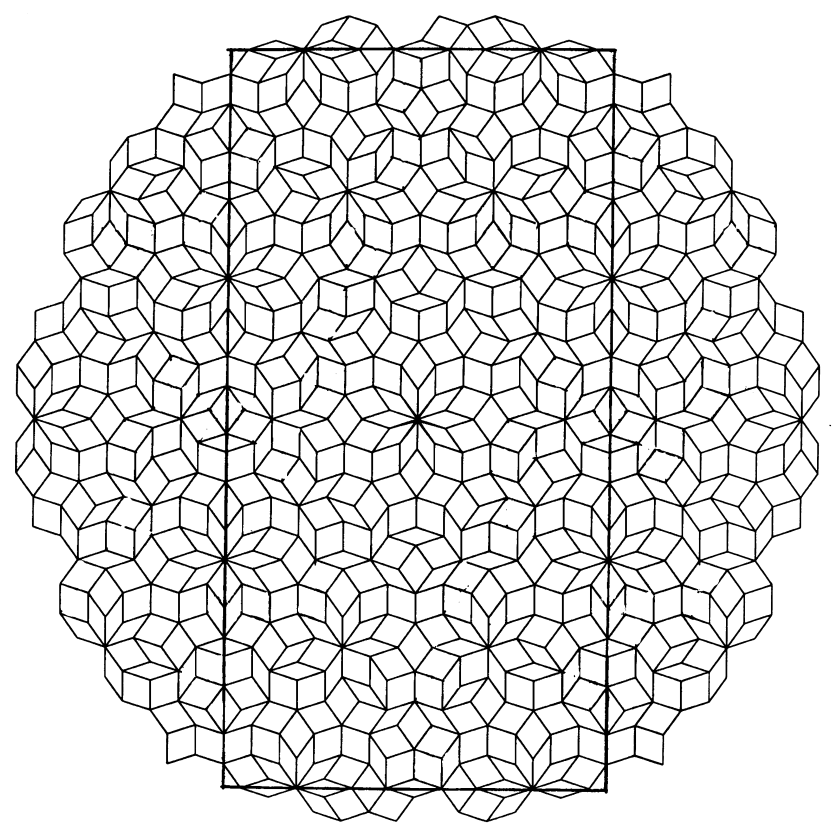

Figure 3. A periodic tiling by Penrose rhombs: a $2 / 1,5 / 3$ approximant in scheme I, with a rectangular unit cell indicated.

$$
\frac{a}{c}=\frac{\tau^{2 n_{1}-n_{2}-1} \sqrt{5}}{\beta}
$$

For scheme II the periods are

$$
\begin{aligned}
& 5 p_{1} \mathbf{a}_{1}+5 q_{1}\left(\mathbf{a}_{2}+\mathbf{a}_{5}\right), \\
& q_{2}\left(\mathbf{a}_{3}-\mathbf{a}_{4}\right)+p_{2}\left(\mathbf{a}_{2}-\mathbf{a}_{5}\right),
\end{aligned}
$$

giving, for the Fibonacci approximants, periods

$$
\begin{aligned}
& 5 \tau^{n_{1}-1} \mathbf{a}_{1} \\
& \tau^{n_{2}}\left(\mathbf{a}_{3}-\mathbf{a}_{4}\right)
\end{aligned}
$$

and

$$
\frac{a}{c}=\frac{5 \tau^{n_{1}-n_{2}-1}}{\beta} .
$$

In both schemes the resulting periodic tiling pattern is orthorhombic, with periods along the two orthogonal directions $\mathbf{a}_{1}$ and $\mathbf{a}_{3}-\mathbf{a}_{4}$ (figure 3 ). It is easily verified that, in scheme I, the sum of the two periods can be divided by 2 to give another period if and only if $p_{1}+q_{2}$ and $p_{2}+q_{1}$ are both even. The orthorhombic unit cell is centred in these cases. In scheme II we get centred orthorhombic cells if and only if $p_{2}$ is even and $p_{1}, q_{1}, q_{2}$ are odd. Figures 4-6 illustrate 1/1, 1/1 and 2/1, 2/1 in scheme I and 1/1, 2/1 in scheme II.

Approximants can also be produced that have periods along $\mathbf{a}_{1}$ and $\mathbf{a}_{2}$ or along $\mathbf{a}_{1}$ and $\mathbf{a}_{3}$. A method for obtaining the appropriate matrices $B$ for these and other cases was given by Ishii (1991). For example, taking

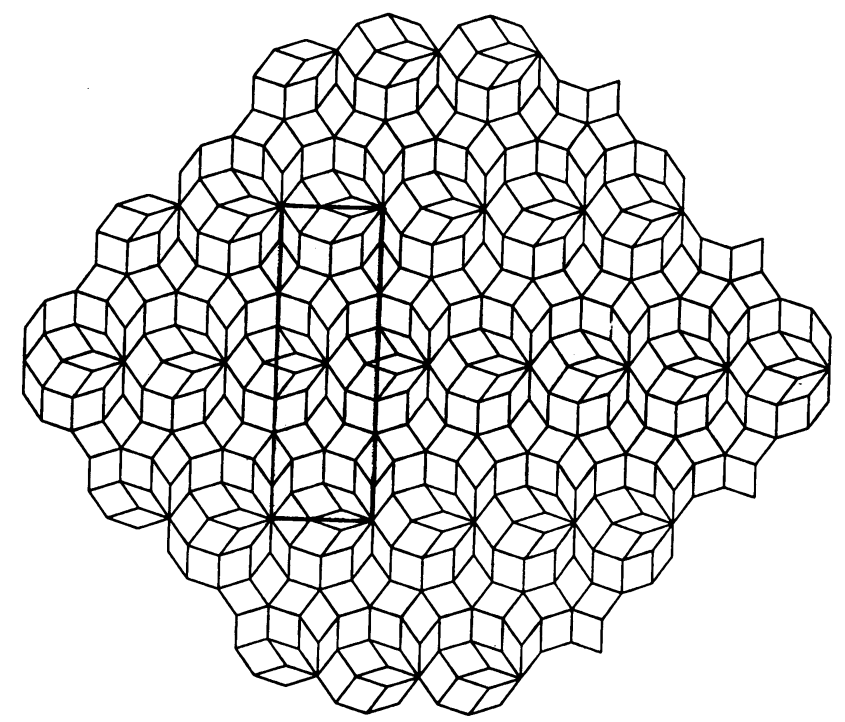

Figure 4. A $1 / 1,1 / 1$ approximant, in scheme I. The orthorhombic unit cell is centred. 


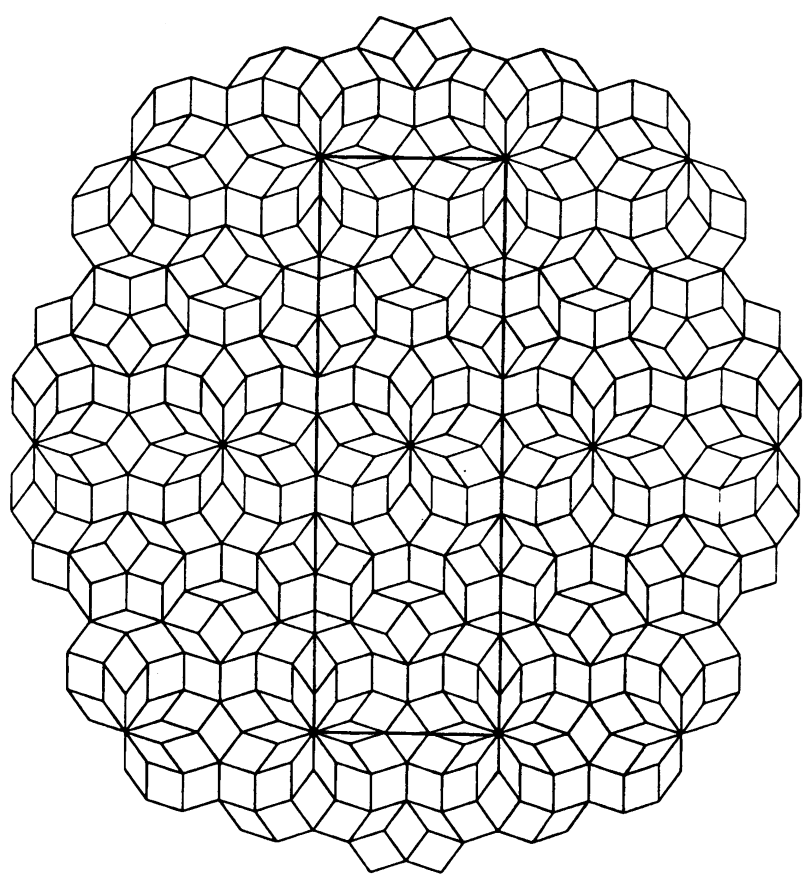

Figure 5. A 2/1, 2/1 approximant, in scheme I. The orthorhombic unit cell is centred.

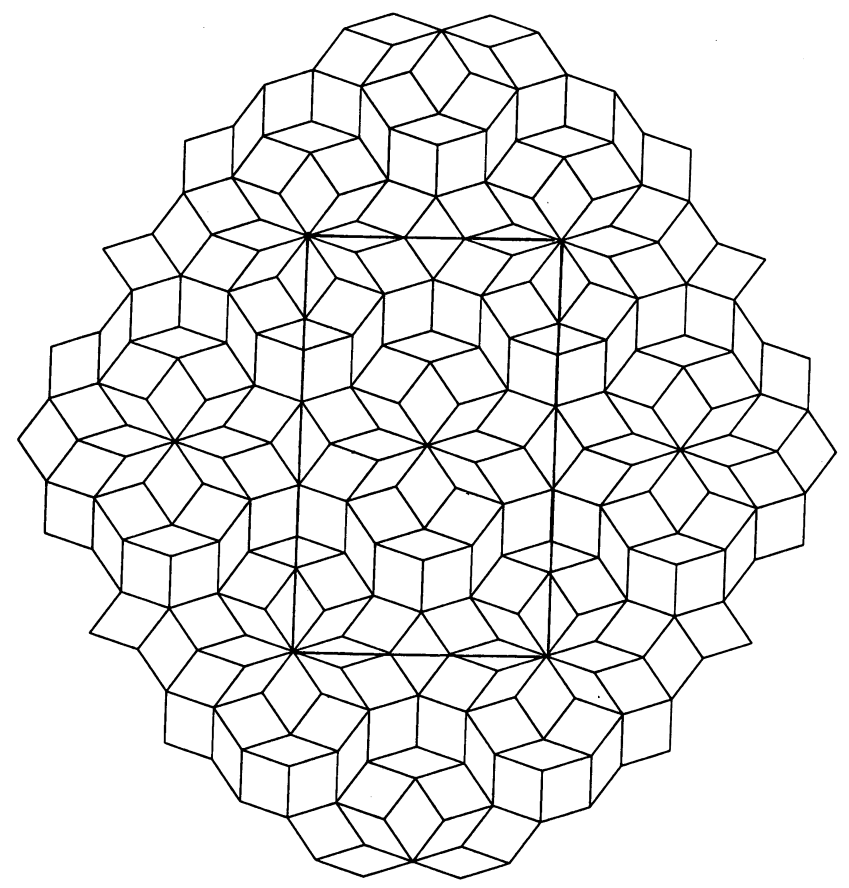

Figure 6. A 1/1, 2/1 approximant, in scheme II. The orthorhombic unit cell is centred.

$$
B=\left(\begin{array}{ccccc}
q & 0 & -q & p & -p \\
0 & q & -p & p & -q \\
1 & 1 & 1 & 1 & 1
\end{array}\right),
$$

we find periods $5 \tau^{n-1} \mathbf{a}_{1}$ and $5 \tau^{n-1} \mathbf{a}_{1}$, suggesting a primitive unit cell in the shape of a Penrose tile. However, a

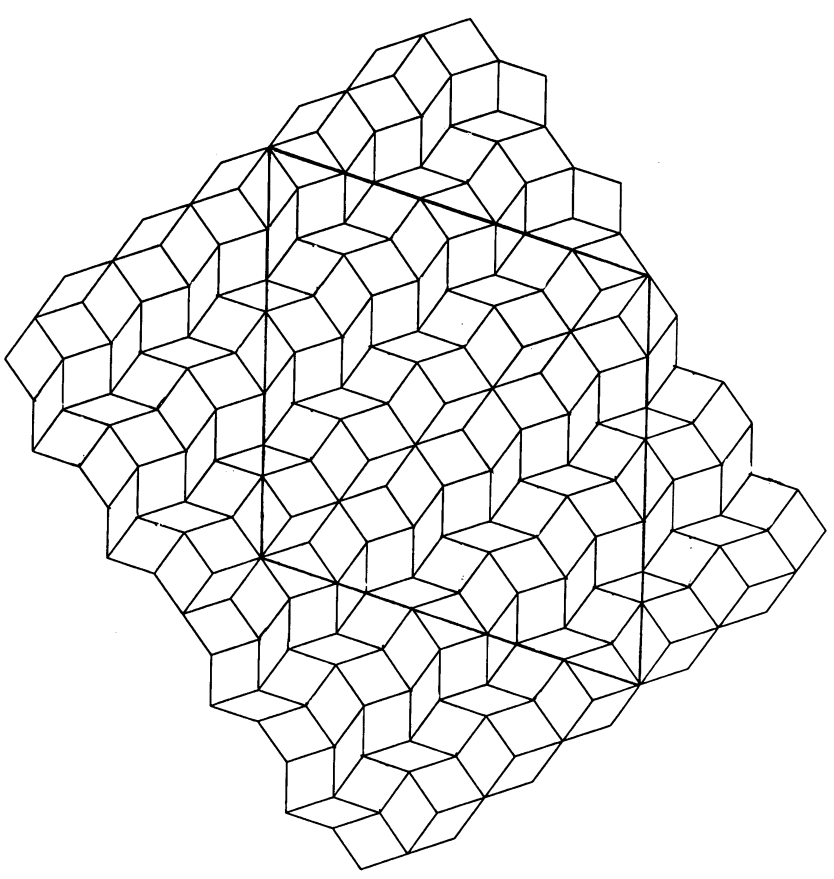

Figure 7. An approximant with equal periods along $\mathbf{e}_{1}$ and $\mathbf{e}_{2}$. Observe the small period $\mathbf{e}_{3}-\mathbf{e}_{5}$, one fifth of the diagonal of the rhombic cell.

shorter period $\tau^{n-1}\left(\mathbf{a}_{1}-\mathbf{a}_{2}\right)$ exists in all these cases. This is readily apparent in figure 7 , which illustrates the simple case of $n=2: p=q=1$.

\section{Acknowledgements}

We wish to thank Anandh Subramaniam for helpful support. Financial support of the Department of Science and Technology, New Delhi, and the Office of Naval Research under Indo-US Cooperative project N00014-951-0095 (Quasicrystals and Crystalline Interfaces) is gratefully acknowledged.

\section{References}

Conway J H and Knowles K M 1986 J. Phys. A: Math. Gen. 19 3645

Conway J H and Sloane N J A 1988 Sphere packings, lattices and groups (London: Springer) p. 120

Ishii Y 1991 Proceedings of the China-Japan seminars: Quasicrystals (eds) K H Kuo and T Ninomiya (Singapore: World Scientific) p. 197

Katz A and Duneau M 1986 J. Phys. 47181

Kramer P and Neri R 1984 Acta Crystallogr. A40 580

Lord E A, Sen S K and Venkaiah V Ch 1990 Simulation 54239

Levine D and Steinhardt P J 1984 Phys. Rev. Lett. 532477

Sadoc J F and Mosseri R 1988 Quasicrystalline materials (eds) C Janot and J M Dubois (Singapore: World Scientific) p. 215

Schechtman D, Blech I, Gratias D and Cahn J W 1984 Phys. Rev. Lett. 531951

Vogg U and Ryder P L 1996 J. Non-Cryst. Solids 194135 